\title{
ON THE CHROMATIC NUMBER OF SUBGRAPHS OF A GIVEN GRAPH
}

\author{
V. RÖDL
}

\begin{abstract}
It is shown that for arbitrary positive integers $m, n$ there exists a $\phi(m, n)$ such that if $\chi(G)>\phi(m, n)$, then $G$ contains either a complete subgraph of size $m$ or a subgraph $H$ with $\chi(H)=n$ containing no $C_{3}$. This gives an answer to a problem of Erdös and Hajnal.
\end{abstract}

Introduction. In [1], [2], [3] P. Erdös raised the following question: Is it true that to every $n$ there is an $f(n)$ such that if $\chi(G) \geqslant f(n)$ then $G$ contains a subgraph $H$ in the sense defined below such that $\chi(H)=n$ and $H$ contains no $C_{3}$ (the circuit of size 3) ? In this paper we prove this conjecture.

We prove the following

THEOREM. For arbitrary positive integers $m, n$ there exists $a \phi(m, n)$ such that if $\chi(G)>\phi(m, n)$ then $G$ contains either a complete subgraph $K_{m}$ of size $m$ or a subgraph $H$ with $\chi(H) \geqslant n$ containing no $C_{3}$.

The conjecture of Erdös and Hajnal now follows easily. Tutte proved [5] that there exists a graph $H$ with $\chi(H)$ arbitrarily large, which contains no $C_{3}$. Now given a graph $G_{1}$ with $\chi(G) \geqslant \phi(m, n)$, then either $G$ contains $K_{m}$ and we find a triangle-free subgraph of $K_{m}$ with big chromatic number, or $G$ contains $H$ as above. We make no attempt here to find the smallest $\phi(m, n)$ with the desired property.

Notation. Given a graph $G=(V, E)$ we shall sometimes write $V=V(G)$, $E=E(G) . H$ is a subgraph of $G$ if $V(H) \subset V(G)$ and $E(H) \subset E(G)$. We shall denote this fact by $H \subset G$. Let $G$ be a graph and let the set $V(G)$ be linearly ordered by $<$. By a left neighborhood of a vertex $v$ we shall understand the graph $L(v, G)$ where

$$
\begin{aligned}
& V(L(v, G))=\left\{v^{\prime} ; v^{\prime}<v,\left\{v^{\prime}, v\right\} \in E(G)\right\}, \\
& E(L(v, G))=E(G) / V(L(v, G)) .
\end{aligned}
$$

Proof of THE Theorem. In the proof we use the following proposition of Zykov [4]. Let $G$ be a graph with $\chi(G) \geqslant(p-1)(q-1)+1$. Let $E(G)=$ $E_{1} \cup E_{2}$ be a partition of $E(G)$. Then either $\chi\left(\left(V(G), E_{1}\right)\right) \geqslant p$ or $\chi\left(\left(V(G), E_{2}\right)\right) \geqslant q$. (This follows since $p-1$ coloring and $\mathrm{a}(q-1)$ coloring

Received by the editors April 6, 1976.

AMS (MOS) subject classifications (1970). Primary $05 \mathrm{C} 15$.

Key words and phrases. Graph, subgraph, triangle-free graph, chromatic number.

O American Mathematical Society 1977 
of the blocks together yield a $(p-1)(q-1)$ coloring of the whole by pairs of colors.)

Obviously we can choose $\phi(2, n)=2$ for every $n$.

Let us suppose that there exists $\phi(m-1, n)$ i.e., every graph $G$ with $\chi(G) \geqslant \phi(m-1, n)$ contains either $K_{m-1}$ or triangle-free $H$ with $\chi(H) \geqslant n$. Let us consider a graph $G_{0}$ with

$$
\chi\left(G_{0}\right)=(n-1)^{\phi(m-1, n)-1}+1=\phi(m, n)
$$

and with $K_{m} \not \subset G_{0}$. Let $<$ be a linear order of $V\left(G_{0}\right)$. Since $K_{m} \not \subset G_{0}$ we have $K_{m-1} \not \subset L\left(v, G_{0}\right)$ for every $v \in V\left(G_{0}\right)$.

If $\chi\left(L\left(v, G_{0}\right)\right)>\phi(m-1, n)$ for some $v \in V\left(G_{0}\right)$, we get (using the induction hypothesis) the existence of a triangle-free graph $H, H \subset L\left(v, G_{0}\right) \subset G_{0}$, with $\chi(H) \geqslant n$. Let us suppose now that $\chi\left(L\left(v, G_{0}\right)\right) \leqslant \phi(m-1, n)-1$ for every $v \in V\left(G_{0}\right)$. Let $\cup_{i=1}^{k} B_{i}^{v}$ be a coloring of the graph $L\left(v, G_{0}\right) ; k<$ $\phi(m-1, n)-1$. Let us define a partition

$$
\begin{aligned}
E\left(G_{0}\right) & =E_{1} \cup E_{2} \cup \cdots \cup E_{\phi(m-1, n)-1}, \\
E_{i} & =\left\{\left\{v_{i}, v\right\} ; v_{i} \in B_{i}^{v}, v \in V\left(G_{0}\right)\right\} .
\end{aligned}
$$

From Zykov's proposition there exists an $i$ such that $\chi\left(V, E_{i}\right) \geqslant n$. Obviously, $\left(V, E_{i}\right)$ is a triangle-free graph.

\section{REFERENCES}

1. P. Erdös, Problems and results in chromatic graph theory, Proof Techniques in Graph Theory, Academic Press, New York 1969, pp. 27-35. MR 40 \#5494.

2. Some unsolved problems in graph theory and combinatorial analysis, Combinatorial Mathematics and Its Applications (Proc. Conf., Oxford, 1969), Academic Press, London, 1971, pp. 97-109. MR 43 \#3125.

3. A. Hajnal, R. Rado and V. T. Sós (Editors), Infinite and finite sets, (Proc. Colloq., Készthely, 1973, dedicated to P. Erdös), North-Holland, Amsterdam, 1975. MR 50 \# 12526.

4. O. Ore, Theory of graphs, Amer. Math. Soc. Colloq. Publ., vol. 38, Amer. Math. Soc., Providence, R. I., 1962. MR 27 \# 740.

5. W. Tutte (under pseudonym Blanche Descartes), A three-colour problem, Eureka, April 1947; Solution, March 1948; Solution to Advanced Problem No. 4526, Amer. Math. Monthly 61 (1954), 352.

Faculty of Nuclear Engineering, CVUT, Department of Mathematics, 11000 Prahal, Husova 5, Czechoslovakia 